Thorben Ziemer*, Dennis Leitz, John Nijim und Christian Rembe

\title{
Fertigung polymerer optischer Phasenplatten zur Erzeugung von Ringmoden mittels direkter Laserlithografie
}

Fabrication of polymeric optical phase plates with direct laser lithography for the creation of doughnut modes

\section{DOI 10.1515/teme-2020-0014}

Zusammenfassung: In diesem Beitrag präsentieren wir die Fertigung polymerer, diskret gestufter spiraler optischer Phasenplatten (SPPs) für die Erzeugung von Ringmoden in Laserstrahlen. Wir beschreiben das von uns verwendete Modell um die Wirkung von SPPs im Fernfeld abhängig von ihrer Geometrie zu simulieren und diese zu dimensionieren. Weiter berichten wir von der Fertigung zunächst 3- und 4-stufiger SPPs aus dem Negativlack AZ nLOF 2070 auf Kalk-Natron-Glas durch Spin Coating und Polymerisation mittels direkter Laserlithografie. Wir zeigen die Wirkung der Bauteile auf das Strahlprofil eines Helium-Neon-Lasers im Fernfeld und validieren damit auch das verwendete Modell.

Schlüsselwörter: Spirale optische Phasenplatte, direkte Laserlithografie, polymere optische Bauteile, Nanoskopie.

Abstract: In this contribution, we present the fabrication of polymeric, discretely stepped spiral optical phase plates (SPPs) for the creation of doughnut modes in laser beams. For this purpose, we describe the model we use to simulate the effect of SPPs in the far field as a function of their geometry and to design such elements. Furthermore, we report on the production of 3- and 4-layered SPPs from the negative resin AZ nLOF 2070 on soda-lime glass via spin coating and polymerization with direct laser lithography. We show the effect of these components on the beam profile of a helium-neon laser in the far field and thereby also validate the model.

Keywords: Spiral phase plate, direct laser lithography, polymeric optical elements, nanoscopy.

\footnotetext{
*Korrespondenzautor: Thorben Ziemer, Institut für Elektrische Informationstechnik, TU Clausthal, Leibnizstraße 28, 38678 Clausthal-Zellerfeld, E-Mail: ziemer@iei.tu-clausthal.de John Nijim, Ohio State University, Columbus, OH 43210, USA Dennis Leitz, Christian Rembe, Institut für Elektrische Informationstechnik, TU Clausthal
}

\section{Einleitung}

In diesem Beitrag beschreiben wir erste Ergebnisse der Fertigung diskret gestufter spiraler optischer Phasenplatten (SPPs) aus Polymeren mittels direkter UV-Laser-Lithografie für die Erzeugung von Laser-Ringmoden.

Es ist hinlänglich bekannt, dass ein Laserstrahl so geformt werden kann, dass sein Modenprofil die Form eines Rings annimmt, d.h. mit einer Intensität, die zum Zentrum des Strahls hin gegen Null geht. Der Strahl bildet dabei einen sogenannten optischen Vortex; diese spezielle Variante einer transversalen elektromagnetischen Mode $\left(\mathrm{TEM}_{0 \mathrm{i}^{*}}\right)$ wird aufgrund ihrer Form auch als DoughnutMode bezeichnet. [1, 2]

Die Verwendung solcher Strahlprofile ist vielfältig. Für uns von besonderem Interesse sind Anwendungen in der STED- und AMI-Nanoskopie (STED: Stimulated Emission Depletion; AMI: Absorbance-Modulation Imaging), bei denen der Bereich im Inneren der Ringmode durch einen separaten Laserstrahl illuminiert wird [5, 6].

Verschiedene Methoden zur Erzeugung von Ringmoden sind bekannt, z.B. Spatial Light Modulators [1, 4], helikale sowie verformbare Spiegel [10] und Mode Converters [3]. Wir beschreiben hier die Verwendung spiraler optischer Phasenplatten (SPPs). Diese verzögern durch ihre über dem Umfangswinkel variierende Dicke ein Laserstrahlenbündel lokal so, dass es in seiner Mitte mit sich selbst destruktiv interferiert [5]. Die resultierende Phasensingularität manifestiert sich als Gebiet geringer Intensität. [2]

Das Fertigen von SPPs ist aufgrund der erforderlichen Präzision nach wie vor aufwändig und teuer. Speziell die Verwendung von Polymeren verspricht eine kostengünstige und enorm flexible Alternative zu sein. Verschiedene Verfahren dazu werden in der Literatur bereits beschrieben. So konnten schon [2] eine SPP aus PMMA (Polymethylmethacrylat) durch zerspanende Bearbeitung fertigen. [8] 
nutzen dagegen einen Abformprozess ähnlich wie in der Linsenherstellung. [7] arbeiten mit PMMA und Elektronenstrahllithografie. Neueste Forschungen wenden auch Zwei-Photonen-Polymerisation an [12].

In der hier vorgestellten Arbeit wird die Möglichkeit untersucht, statische SPPs für den Einsatz im Labor flexibel mittels direkter UV-Laser-Lithografie herzustellen. Wir beschreiben zunächst ein Modell, anhand dessen wir SPPs simulieren und deren Geometrie entwerfen. Für experimentelle Nachweise fertigen wir 3- und 4-stufige SPPs, indem auf ein Glassubstrat schichtweise ein negativer Fotolack aufgebracht wird, gefolgt von lokaler Polymerisation durch Belichtung und nasschemischer Entwicklung. Anhand einer Untersuchung der Wirkung dieser Elemente auf das Profil eines Helium-Neon-Lasers (He-Ne-Laser) bewerten wir den Ansatz hinsichtlich seiner Machbarkeit, beurteilen den Fertigungsprozess und validieren gleichzeitig das Modell.

Wir können zeigen, dass Polymere auch in Kombination mit direkter Laser-Lithografie grundsätzlich für SPPs geeignet sind und dass dies ein vielversprechender Ansatz ist, genau angepasste optische Elemente für verschiedene Wellenlängen und Wellenfronten zu fertigen.

\section{Methoden}

Um SPPs für die Fertigung zu dimensionieren und später die Ergebnisse analysieren und bewerten zu können, wählen wir zunächst ein passendes mathematisch-physikalisches Modell. Dieses wird dann als numerische Simulation in MATLAB implementiert.

Zur Modellvalidierung und zum konzeptuellen Nachweis werden diskretisierte SPPs mit $N<5$ Stufen gefertigt und in einem Testaufbau auf ihre Wirksamkeit untersucht.

\subsection{Theorie und Modell}

Das Erzeugen von Ringmoden mittels SPPs folgt dem Grundprinzip, dass Teile eines Laserstrahlenbündels unterschiedlich viel Material durchqueren. Die daraus resultierende winkelpositionsabhängige Verzögerung führt zu unterschiedlichen Phasenverschiebungen, sodass es im Fernfeld nach der SPP im Zentrum des Strahlenbündels zu destruktiver Interferenz kommt, also ein Bereich geringerer Intensität entsteht. Eine Möglicheit ist, mit der SPP die Phasen $\phi$ der an der optischen Achse jeweils einander gegenüberliegenden Strahlen um genau $\Delta \phi=\pi$ zueinander zu verschieben. Abb. 1 verdeutlicht das Prinzip anhand der Draufsicht auf ein Element mit vier Ebenen, deren Höhen so variieren, dass jede Ebene eine um $\Delta \phi=\pi / 2$ größere Phasenverschiebung verursacht. Andere Varianten mit Auslöschung nach mehrfacher Interferenz sind möglich.

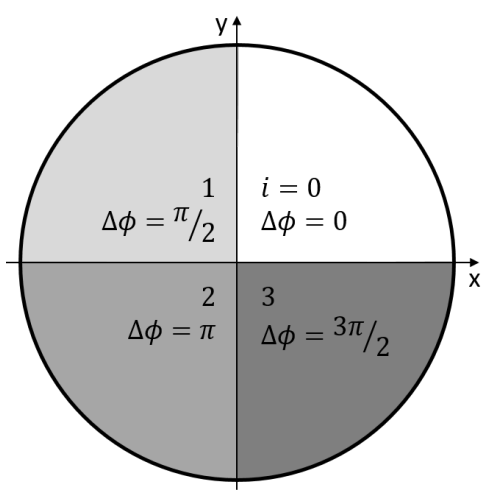

Abb. 1: Beispiel einer vierstufigen spiralen optischen Phasenplatte (SPP; $N=4$ ) in Draufsicht. Tritt ein Strahlenbündel der Wellenlänge $\lambda$ in z-Richtung durch das Zentrum der Platte, so bewirken die einander gegenüberliegenden Ebenen $i=0$ und 2 sowie 1 und 3 jeweils um $\Delta \phi=\pi$ bzw. $\lambda / 2$ zueinander versetzte Phasenverschiebungen, sodass die Strahlen hinter der SPP bei Überlagerung teilweise destruktiv interferieren.

Für die Dimensionierung geeigneter SPPs gehen wir von dem folgenden Modell aus. Dabei beschränken wir uns nach [11] hier auf das Regime der paraxialen Optik sowie das Fernfeld und lassen Effekte durch Polarisation unberücksichtigt. ${ }^{1}$

Wir nehmen zunächst an, dass das einfallende Strahlenbündel als Gauß-Strahl beschrieben werden kann, also auch gut kollimiert ist und einen ausreichend kleinen Durchmesser hat. Dies ist die TEM der Ordnung $00\left(\mathrm{TEM}_{00}\right)$. An einer Position $z$ entlang der optischen Achse sei dessen Radius $w_{0}=w(z)$. Die komplexe Amplitude $u$ des Strahls hat dann in Polarkoordinaten $r$ und $\varphi$ näherungsweise das Profil

$$
u(r, \varphi) \propto e^{-\left(r / w_{0}\right)^{2}} .
$$

Ziel ist die Erzeugung sogenannter Ring- oder DoughnutModen. Diese entstehen aus Strahlen, deren Moden mathematisch mittels Laguerre-Polynomen beschrieben und deswegen auch als Laguerre-Gauß-Moden (LG-Moden) bezeichnet werden. Für das Profil $u^{\prime}$ dieser LG-Moden (auch $\mathrm{TEM}_{0 \mathrm{i}} *$ als spezielle Form der TEM) ergibt sich $[2,7,9]$

$$
u^{\prime}(r, \varphi) \propto e^{-\left(r / w_{0}\right)^{2}} \cdot e^{-i \ell \varphi}
$$

1 Siehe dazu weiterführend bspw. [2]. 
Dieses Profil führt im Fernfeld zu jener Interferenz, die in einer in der Fläche ringförmig verteilten Strahlungsintensität resultiert.

Ein Vergleich der beiden Amplitudenprofile zeigt, dass die erforderliche Operation von (1) zu (2) interpretiert werden kann als eine in Umfangsrichtung vorgenommene Phasenvariation des ursprünglichen Strahlenbündels. Diese kann durch eine SPP erzeugt werden [11]. Der ganzzahlige, positive Faktor $\ell$ bezeichnet dabei die topologische Ladung (topological charge) und ergibt sich aus der Anzahl der Phasendrehungen innerhalb einer Wellenlänge. Für diese Arbeit werden lediglich Strahlen mit $\ell=1$ betrachtet.

Voraussetzung für das Auftreten eines Ringprofils ist destruktive Interferenz der Strahlen nahe der optischen Achse. Vollständige Auslöschung tritt dann auf, wenn zwei Strahlen interferieren, die einen Phasenversatz von exakt $\Delta \phi=\pi$ (oder ungerade Vielfache davon) aufweisen. Um für alle Strahlen nahe der optischen Achse durch das Zentrum der SPP zu erreichen, dass auch ein entsprechend phasenverschobener Strahl existiert, sollen zwei Strahlen der Wellenlänge $\lambda$ bei Durchgang durch zwei um $\varphi=$ $\pi$ zueinander versetzte („einander gegenüberliegende“) Stellen der SPP gerade eine um $\Delta \phi=\pi$ unterschiedliche Phasendrehung erfahren (Abb.1).

Mit den Brechungsindizes der SPP und des umgebenden Mediums, $n_{1}$ und $n_{0}$, muss für diesen Idealfall die Höhe $h$ des Elements also der Funktion

$$
h(\varphi)=\ell \cdot \frac{\varphi}{2 \pi} \cdot \frac{\lambda}{n_{1}-n_{0}}
$$

genügen. Dies ist die Entwurfsgleichung der SPP. [7]

Es ist bekannt, dass das Verhalten einer idealen SPP, mit kontinuierlich in Winkelrichtung zunehmender Dicke, durch diskret gestufte Phasenplatten („Wendeltreppe“) angenähert werden kann [11]. Für das obige Modell ergibt sich beispielsweise mit $N=8$ Stufen bereits ein deutliches Ringprofil. Abb. 2 zeigt die Geometrie und das simulierte Intensitätsprofil eines solchen Elements.

Bei gleichmäßiger Aufteilung sollte die Höhe einer einzelnen Stufe dann die Forderung

$$
\Delta h=\frac{\ell}{N} \cdot \frac{\lambda}{n_{1}-n_{0}}
$$

erfüllen. Daraus abgeleitet ist die angestrebte Gesamtdicke $h_{i}$ einer einzelnen Ebene bzw. Schicht

$$
h_{i}=i \cdot \Delta h, \quad i=0,1, \ldots,(N-1),
$$

wobei $i=0$ die Substratebene bezeichnet und $N$ dementsprechend die Anzahl der Ebenen inklusive Substrat. ${ }^{2}$

2 Anmerkung: Aus der Forderung, dass je genau zwei Ebenen zusammen den erforderlichen Phasenversatz erzeugen, wären nur

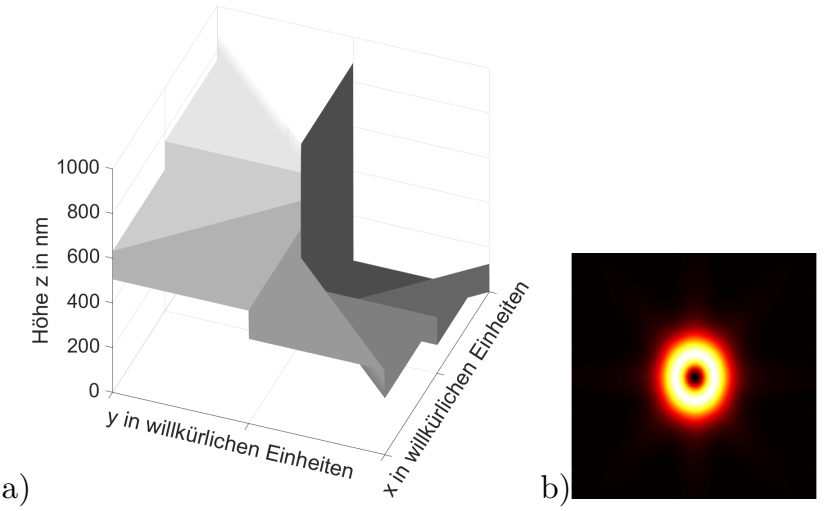

Abb. 2: Simulation einer idealen 8-stufigen SPP. (a) Wendeltreppenstruktur. (b) Intensität im Fernfeld eines Gauß-Strahls nach Durchquerung des Zentrums der SPP.

Für die hier vorgenommenen Analysen wird auf Basis des vorgestellten Modells die Strahlungsintensität im Fernfeld simuliert. Diese ist grundsätzlich proportional zum Quadrat der Amplitude des elektrischen Feldes, welches wiederum mit der FraunhofER-Näherung des Beugungsintegrals approximiert werden kann. Deren Lösung ist die Fouriertransformierte der Aperturfunktion, hier des durch die SPP definierten $u^{\prime}$ (Gleichung (2)). [2, 7, 9]

Für die Simulationen wird dies mit einer 2D FFT von (2) umgesetzt (zweidimensionale schnelle FOuRIERTransformation), dann quadriert. Die Wellenlänge des verwendeten Testlasers wird entsprechend der Herstellerangabe als $\lambda=632,8 \mathrm{~nm}$ angenommen. Das verwendete Polymer (AZ nLOF 2070; s.u.) hat laut Lieferant bei dieser den Brechungsindex $n_{1}=1,626$. Unter Vernachlässigung des Glassubstrats wird für das umgebende Medium $n_{0}=1$ gesetzt.

\subsection{Entwurf von Phasenplatten und Messaufbau}

Anhand der Beziehungen (3) bis (5) werden zunächst 3und 4-stufige SPPs entworfen und dann gefertigt. Tab. 1 in Abschnitt 3 zeigt die Ergebnisse.

Ein experimenteller Nachweis der Wirksamkeit der gefertigten SPPs erfolgt mit einem He-Ne-Laser (Lumentum Model 1103P, Lumentum Operations LLC, San Jose) und dem Aufbau gemäß Abb. 3. Mittels einer Linse wird die Fourierebene der SPP auf den Chip einer CMOSKamera abgebildet. Der Interferenzeffekt kann darüber

geradzahlige $N$ zulässig. Simulationen und Experimente zeigen aber, dass auch für ungerade $N$ Intensitäten nahe Null erreicht werden können. 
hinaus im Fernfeld der SPP mit bloßem Auge beobachtet werden.

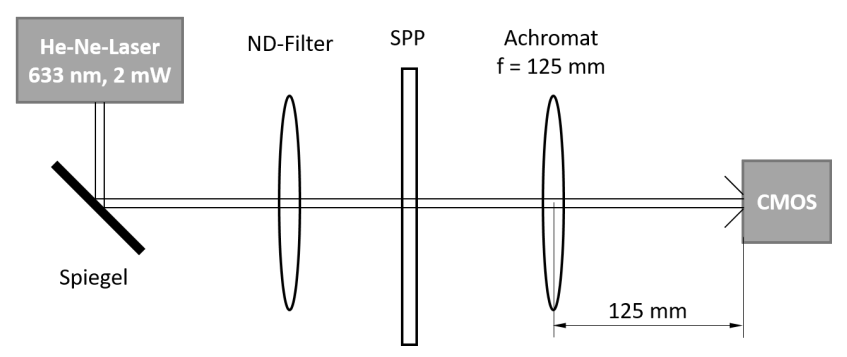

Abb. 3: Messaufbau zur experimentellen Untersuchung der Wirkung von SPPs. Helium-Neon-Laser (He-Ne-Laser) mit $\lambda=632,8 \mathrm{~nm}$, Strahldurchmesser 0,63 $\mathrm{mm}$ ( $\pm 3 \%$ ), Ausgangsleistung $2 \mathrm{~mW}$, linear polarisiert (500:1; Reinheit der TEM 00 $>95 \%$ ), Abschwächung mit Neutraldichtefilter (ND-Filter) und Messung mit CMOS-USB-Kamera.

\subsection{Fertigung mit Laserlithografie}

Hier werden 3- und 4-stufige polymere SPPs gefertigt. Dies erfolgt mit dem industriellen Negativlack AZ nLOF 2070 (auf Basis von Phenolharz), der mit AZ EBR Solvent (auf Basis von 1-Methoxy-2-propylacetat (PGMEA)) verdünnt und dann in mehreren Schichten mittels Spin Coating auf Kalk-Natron-Glas aufgetragen, unter direkter Laserlithografie polymerisiert und mit AZ $826 \mathrm{MIF}$ (2,38\% Tetramethylammoniumhydroxid in $\mathrm{H}_{2} \mathrm{O}$ ) entwickelt wird. Die AZ-Produkte wurden bezogen von der MicroChemicals GmbH, Ulm.

Der Fotolack wird im Verhältnis 1:3 mit dem Verdünner gemischt und zur Verminderung von Luftblasen und Inhomogenitäten für ca. 45 min ruhen gelassen.

Das als Substrat verwendete Glas wird vorbereitet, indem es zunächst nacheinander mit Aceton und dann mit 2-Propanol gespült wird, um organische und anorganische Verunreinigungen zu entfernen. Dann erfolgt zur Desorption ein 20-minütiges Ausheizen bei $200^{\circ} \mathrm{C}$ auf einer Heizplatte, um $\mathrm{H}_{2} \mathrm{O}$ und $\mathrm{OH}$-Bindungen aus der Oberfläche zu lösen.

Nach diesem und allen weiteren Temperschritten ruht die Probe, bis sie wieder auf Raumtemperatur $\left(23^{\circ} \mathrm{C}\right)$ abgekühlt ist.

Als nächstes wird die erste Schicht des Fotolacks erzeugt. Beschichtungen erfolgen mittels Spin Coating, wobei die Schichtdicke maßgeblich von der Viskosität des Lacks und von der Drehzahl abhängt. Der Negativlack wird mit einer Spritze auf das stillstehende Substrat aufgebracht und dann das in Abb. 4 gezeigte Beschichtungsprofil ausgeführt. Die erzielten Schichthöhen können Tab. 1 entnommen werden.

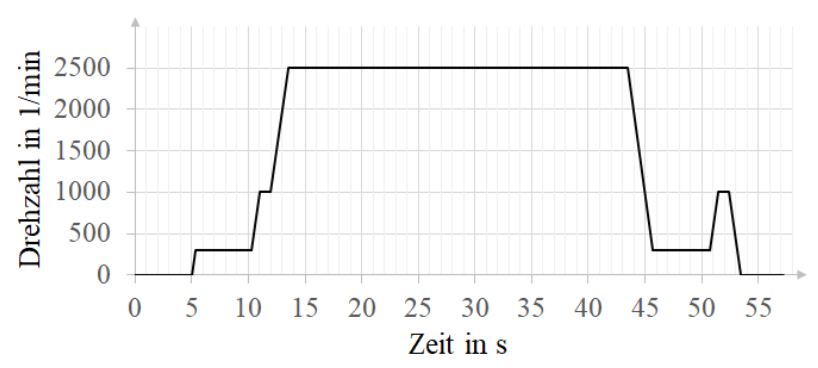

Abb. 4: Drehzahl-Zeit-Diagramm für den Spin-Coating-Prozess. Die Beschleunigung ist jeweils $1000 \frac{1 / \mathrm{min}}{\mathrm{s}}$.

Nach dem Spin Coating wird auf einer Heizplatte bei $110^{\circ} \mathrm{C}$ für $60 \mathrm{~s}$ ein Softbake der Probe durchgeführt, um verbleibendes Lösungsmittel aus dem Lack zu entfernen.

Dann wird sie mit dem Laserlithografiesystem $\mu P G$ 101 belichtet (Hersteller Heidelberg Instruments MiKROTECHNIK GmbH, Heidelberg; Schreibkopf zur lateralen Auflösung von $1 \mu \mathrm{m})$. Die Belichtungsdosis beträgt bei den hier präsentierten SPPs $3979 \mathrm{~mJ} / \mathrm{cm}^{2}$.

Es folgt ein Post Exposure Bake (PEB) auf einer Heizplatte bei $105^{\circ} \mathrm{C}$ für $120 \mathrm{~s}$ zur Fortsetzung der durch die Belichtung begonnenen Quervernetzung des Polymers.

Anschließend wird durch Tauchentwicklung für $25 \mathrm{~s}$ im Entwickler und Spülung mit demineralisiertem Wasser sowie gereinigter Druckluft das unbelichtete Material entfernt. Zur weiteren Erhöhung der Stabilität der Strukturen werden diese für $7 \mathrm{~min}$ bei $160{ }^{\circ} \mathrm{C}$ auf einer Heizplatte gebacken („Hardbake“).

Dann folgt die nächste Schicht nach der gleichen Vorgehensweise exklusive der Reinigung und Desorption.

\section{Ergebnisse und Diskussion}

Eine Messung der Schichthöhen der hier gefertigten SPPs erfolgt mit einem konfokalen Laser-Scanning-Mikroskop (CLSM). Die Ergebnisse zeigen Abb. 5 und Tab.1. Es ergibt sich, dass die Güte der Oberflächen noch nicht ideal ist. Zum einen entsprechen die Höhen nicht den Entwurfsparametern, zum anderen weisen einige Schichten noch markante Riefen auf. Da deren Tiefe mit bis zu $\Delta h_{\text {Riefen }}<100 \mathrm{~nm}$ ähnlich den Stufenhöhen und der Wellenlänge des zu formenden Lasers ist, ist davon auszugehen, dass dies einen Effekt auf die Strahlqualität haben wird. Allerdings zeigt sich, dass es für den hier angestrebten prinzipiellen Nachweis der Funktionalität der SPPs 

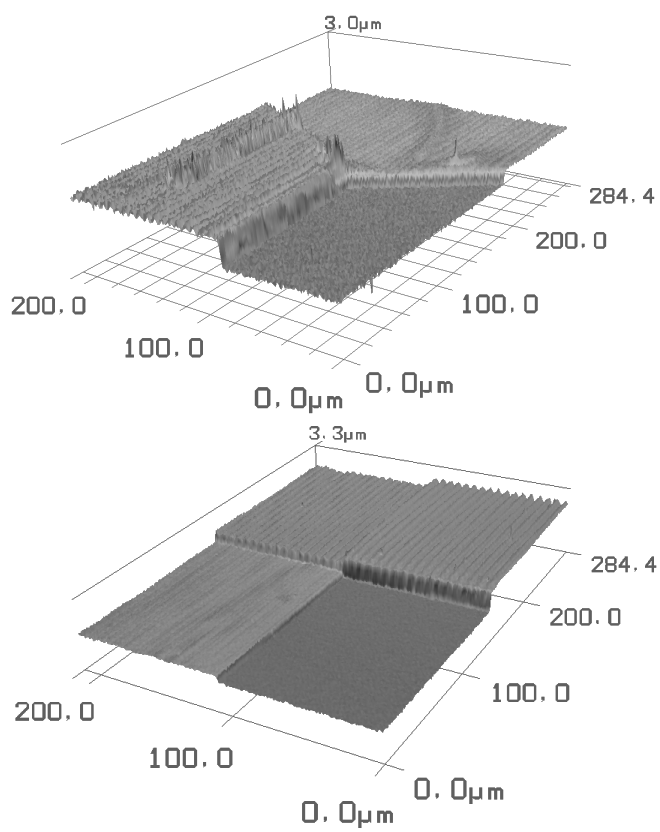

Abb. 5: Höhenprofile gefertigter 3- und 4-stufiger SPPs, gemessen mit einem konfokalen Laser-Scanning-Mikroskop (CLSM).

vernachlässigt werden kann. Eine weitere Optimierung des Fertigungsprozesses wird voraussichtlich auch zu verbesserten Oberflächen führen.

Tab. 1: Vergleich der Designhöhen aus dem Entwurfsmodell mit den mittleren Höhen der gefertigten SPPs.

\begin{tabular}{rllll}
\hline & \multicolumn{4}{c}{ mittlere Schichthöhe $h_{i}$ in $\mathbf{n m}$} \\
& \multicolumn{2}{l}{ 3-stufige SPP } & \multicolumn{2}{l}{ 4-stufige SPP } \\
Schicht-Nr. $\boldsymbol{i}$ & Soll & Ist & Soll & Ist \\
\hline 0 & 0 & 0 & 0 & 0 \\
1 & 337 & 279 & 253 & 302 \\
2 & 674 & 534 & 505 & 732 \\
3 & - & - & 758 & 1133 \\
\hline
\end{tabular}

Mit dem in Abb. 3 dargestellten Testaufbau wird die Wirkung der gefertigten Elemente in ihrer Fourierebene (und damit auch im Fernfeld) untersucht. Abb. 6 und 7 zeigen die nach Simulationen erwarteten Intensitätsprofile je einer idealen 3- und 4-stufigen SPP (a) neben den sich aus diesen Messungen ergebenden Strahlintensitäten (b). Darüber hinaus finden sich dort in (c) die Ergebnisse von Simulationen, bei denen die Stufenhöhen an die tatsächlich gemessenen Höhen angepasst sind.

Die im Experiment erzeugten Strahlprofile entsprechen nicht den simulierten Idealfällen, nähern sich aber bereits deutlich der angestrebten Form mit einem Bereich niedrigerer Intensität im Zentrum des Strahlenbündels, der umgeben ist von Bereichen maximaler Intensität.
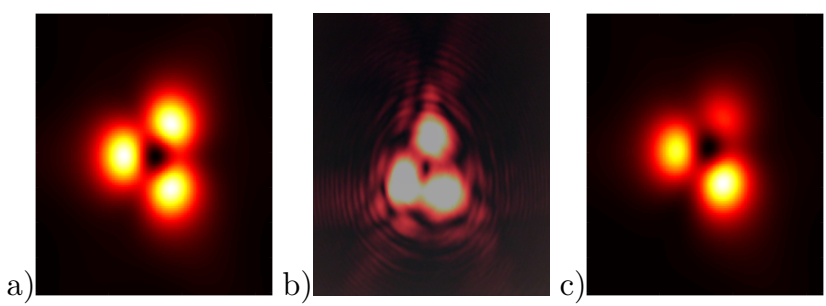

Abb. 6: Simulation einer idealen 3-stufigen SPP (a), Ergebnis der Messung (b) und Simulation mit den realisierten Fertigungsparametern (c).
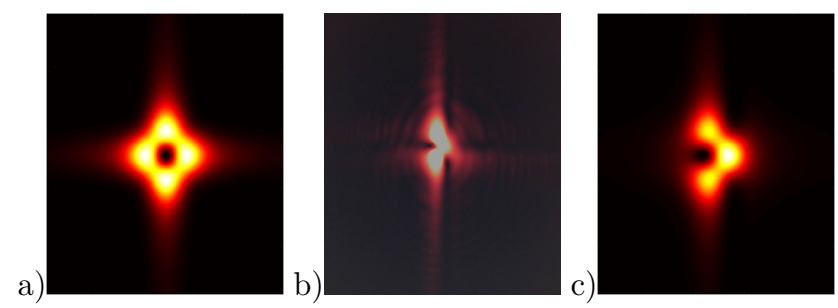

Abb. 7: Simulation einer idealen 4-stufigen SPP (a), Ergebnis der Messung (b) und Simulation mit den realisierten Fertigungsparametern (c).

Werden die gemessenen Stufenhöhen der gefertigten Elemente aus Tab. 1 in das Modell gegeben, so stimmen die simulierten Strahlprofile bereits gut mit den beobachteten überein (Abb. 6c und 7c). Es kann somit gezeigt werden, dass die Abweichung vom Idealfall hauptsächlich in der Abweichung der Stufenhöhen begründet ist. Gleichzeitig validiert dies das Modell.

Tab. 2 listet die in den gefertigten Elementen tatsächlich gemessenen mittleren Stufenhöhen und die sich daraus theoretisch ergebenden Phasenverschiebungen $\Delta \phi$ bezogen auf $\pi$ (berechnet durch Anwendung der Entwurfsgleichung (3) mit $\phi \hat{=} \varphi$ ). Vollständige destruktive Interferenz tritt bei zwei Strahlen für $\Delta \phi / \pi=1$ auf. $^{3}$ Im Falle der 3stufigen SPP zeigt sich bspw., dass die Stufenhöhe $\Delta h_{02}$ gerade ein $\Delta \phi_{02} \approx 1,06 \pi$ hervorruft. Bei diesem Bauteil kann also erwartet werden, dass es einen größeren Bereich nahezu vollständiger Auslöschung im Strahlprofil gibt. Das Ergebnis der Messung bestätigt dies (Abb.6b).

Die hier gefertigten SPPs liefern bereits gute Ergebnisse. Die mit ihnen erzeugten Strahlprofile entsprechen dem eingeführten Modell und zeigen selbst bei einer relativ geringen Anzahl Stufen und trotz der noch nicht idealen Stufenhöhen eindeutig in ihren Zentren Bereiche niedriger Lichtintensität. Es steht zu erwarten, dass durch Erhöhung der Stufenzahl und Korrektur der Stufenhöhen ein ideales Ringprofil weiter angenähert werden kann. Entscheidend

3 Bei Mehrfachüberlagerung sind auch andere Werte möglich. 
Tab. 2: Höhen $\Delta h_{j k}=\left|h_{k}-h_{j}\right|$ der einzelnen Stufen bei den gefertigten SPPs und theoretisch daraus resultierende Phasenverschiebungen $\Delta \phi_{j k}=\left|\phi_{k}-\phi_{j}\right|$ eines Gauß-Strahls.

\begin{tabular}{|c|c|c|c|c|c|}
\hline \multirow[b]{4}{*}{$k$} & \multirow[b]{4}{*}{$j$} & \multicolumn{2}{|c|}{ 3-stufige SPP } & \multicolumn{2}{|c|}{ 4-stufige SPP } \\
\hline & & $\Delta h_{j k}$ & $\Delta \phi_{j k}$ & $\Delta h_{j k}$ & $\Delta \phi_{j k}$ \\
\hline & & $\mathbf{n m}$ & $\pi$ & $\mathbf{n m}$ & $\pi$ \\
\hline & & & & & \\
\hline 0 & 0 & 0 & 0 & 0 & 0 \\
\hline 0 & 1 & 279 & 0,55 & 302 & 0,60 \\
\hline 0 & 2 & 534 & 1,06 & 732 & 1,45 \\
\hline 0 & 3 & - & - & 1133 & 2,24 \\
\hline 1 & 2 & 255 & 0,50 & 430 & 0,85 \\
\hline 1 & 3 & - & - & 831 & 1,64 \\
\hline 2 & 3 & - & - & 401 & 0,79 \\
\hline
\end{tabular}

ist also zunächst eine weitere Optimierung der Fertigung, um damit eine genauere Einstellung der erzeugten Schichthöhen sowie der Oberflächengüte zu gewährleisten.

\section{Zusammenfassung und Ausblick}

In diesem Beitrag demonstrieren wir den Entwurf und die Fertigung diskret gestufter spiraler optischer Phasenplatten aus Polymeren, um damit Laserstrahlen zu Ringmoden zu formen. Wir zeigen, dass solche SPPs durch direkte Laserlithografie mit preisgünstigen Materialien aus handelsüblichen Polymeren hergestellt und mit einem Laserstrahl der Designwellenlänge, einer Linse und einem digitalen Bildsensor im Fokus der Linse einfach geprüft werden können. Mit den gefertigten SPPs modifizieren wir den Gauß-Strahl eines He-Ne-Lasers und erreichen die angestrebte Ringmode bereits in erster Näherung.

Darüber hinaus können wir für unsere Arbeit ein Modell des Verhaltens der SPP in MATLAB implementieren und erfolgreich erste Validierungen durchführen. Der Einfluss der SPP auf das Fernfeld eines Laserstrahls, der diese durchstrahlt, stimmt mit unserer Simulation der Fraunhofer-Beugung desselben überein. Somit kann unser Modell für den Entwurf der Schichthöhen auch für andere Wellenlängen verwendet werden.

Dieses Modell wird als Grundlage dienen, um nach Ergänzung weiterer relevanter Aspekte das Systemverhalten zukünftig noch präziser abzubilden. Dabei werden die Modellierung des Nahfelds unter umfassenderer Berücksichtigung von Beugung und hinsichtlich der Polarisation des Strahlenbündels als nächstes im Fokus stehen.

Die von uns beschriebenen Fertigungstechniken zeigen großes Potential, in Zukunft verschiedene Forschungsprojekte der optischen Messtechnik durch die Bereitstellung spezialisierter optischer Bauteile unterstützen zu können.
Für die hier vorgestellten SPPs wird zunächst eine weitere Optimierung des Fertigungsprozesses erfolgen, insbesondere zur genaueren Einstellung der Schichtdicken und Verbesserung der Oberflächengüte.

Weitere Experimente zur Bewertung des Verhaltens und der Wirkung der SPPs bei UV-Strahlung werden folgen. Dies ist für ihre Anwendung zur Nanoskopie besonders relevant. Dabei sind neben der Qualität der erzeugbaren Modenprofile vor allem auch die Transmissivität und Beständigkeit der verwendeten Polymere von Interesse.

\section{Literatur}

[1] D. L. Andrews. Structured light and its applications: An introduction to phase-structured beams and nanoscale optical forces. Academic, Amsterdam and Boston, 2008.

[2] M. W. Beijersbergen, R. Coerwinkel, M. Kristensen und J. P. Woerdman. Helical-wavefront laser beams produced with a spiral phaseplate. Optics Communications, 112(5-6):321-327, 1994.

[3] J. Courtial und M. J. Padgett. Performance of a cylindrical lens mode converter for producing laguerre-gaussian laser modes. Optics Communications, 159(1-3):13-18, 1999.

[4] J. E. Curtis und D. G. Grier. Modulated optical vortices. Optics Letters, 28(11):872-874, 2003.

[5] T. A. Klar, S. Jakobs, M. Dyba, A. Egner und S. W. Hell. Fluorescence microscopy with diffraction resolution barrier broken by stimulated emission. Proceedings of the National Academy of Sciences of the United States of America, 97 (15):8206-8210, 2000.

[6] R. Kowarsch, C. Geisler, A. Egner und C. Rembe. Superresolution reflection microscopy via absorbance modulation: a theoretical study. Optics Express, 26(5):5327-5341, 2018.

[7] M. Massari, G. Ruffato, M. Gintoli, F. Ricci und F. Romanato. Fabrication and characterization of high-quality spiral phase plates for optical applications. Applied Optics, 54(13): 4077, 2015.

[8] S. S. R. Oemrawsingh, E. R. Eliel, J. P. Woerdman, E. J. K. Verstegen, J. G. Kloosterboer und G. W. t. Hooft. Halfintegral spiral phase plates for optical wavelengths. Journal of Optics A: Pure and Applied Optics, 6(5):S288-S290, 2004.

[9] B. Sephton, A. Dudley und A. Forbes. Revealing the radial modes in vortex beams. Applied Optics, 55(28):7830-7835, 2016.

[10] R. K. Tyson, M. Scipioni und J. Viegas. Generation of an optical vortex with a segmented deformable mirror. Applied Optics, 47(33):6300-6306, 2008.

[11] T. Watanabe, M. Fujii, Y. Watanabe, N. Toyama und $\mathrm{Y}$. Iketaki. Generation of a doughnut-shaped beam using a spiral phase plate. Optical Engineering, 75(12):5131-5135, 2004.

[12] H. Wei, A. K. Amrithanath und S. Krishnaswamy. 3d printing of micro-optic spiral phase plates for the generation of optical vortex beams. IEEE Photonics Technology Letters, 31(8): 599-602, 2019. 